\title{
Evaluación de sostenibilidad agroecológica de dos experiencias de producción con énfasis en hortalizas, Venezuela
}

\section{Agroecological sustainability assessment of two production systems with an emphasis on vegetables in Venezuela}
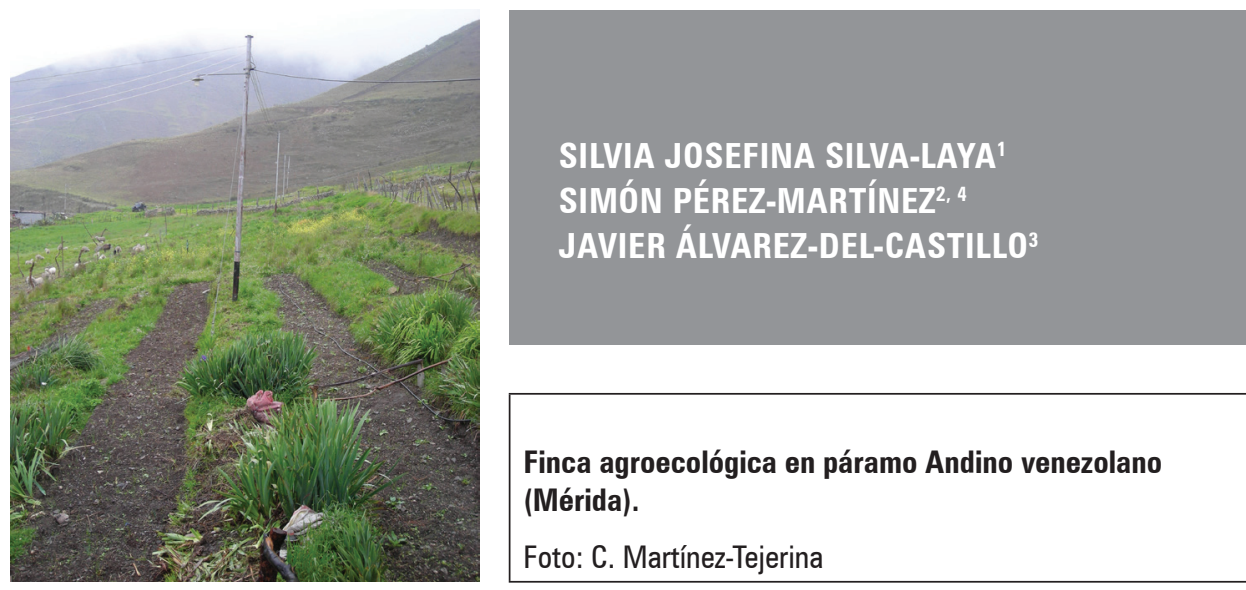

\section{RESUMEN}

Esta investigación tuvo como objetivo evaluar la sostenibilidad de dos sistemas de producción agroecológica de hortalizas y plantas medicinales, en una zona periurbana (NUDES-La Limonera) y agrícola (FIRP) de Venezuela. Se realizaron observaciones descriptivas durante once meses entre 2014-2015. Se aplicó un cuestionario sociodemográfico; y se cuantificaron dieciséis indicadores de sostenibilidad en las dimensiones ecológica (IE), económica (IK) y sociocultural (ISc) mediante metodologías publicadas anteriormente. Se encontró que El NUDES-La Limonera forma parte de un proyecto político del Ejecutivo Nacional y sus indicadores de sostenibilidad alcanzaron niveles menos favorables, especialmente algunos IE (conservación de la vida en el suelo y dependencia de insumos externos) y IK (autosuficiencia alimentaria, papel del trabajo familiar, riesgo económico y diversidad de productos para la venta). Los ISc muestran mayor fortaleza, particularmente en la densidad de relaciones con otros colectivos, sin embargo, la satisfacción de necesidades básicas como la vivienda no fue satisfactorio ya que la tierra no es propia (solo la guardia y custodia). La FIRP forma parte de una iniciativa de productores organizados para la diversificación agrícola de los Andes venezolanos. Presentó los valores más favorables a la sostenibilidad en las tres dimensiones. Vale destacar que IK, la productividad

1 Universitat Politècnica de Catalunya, Sostenibilidad, Tecnología y Humanismo, Barcelona (España). ORCID SilvaLaya, S.J.: 0000-0003-1049-4318

2 Universidad. Estatal de Milagro (UNEMI), Facultad de Ciencias de la Ingeniería, Cantón Milagro (Ecuador). ORCID Pérez-Martínez, S.: 0000-0001-7349-7036

3 Universitat Politècnica de Catalunya, Cátedra UNESCO de Sostenibilitat, Terrassa (España). ORCID Álvarez-DelCastillo, J.: 0000-0002-5640-5176

4 Autor para correspondencia. sperezm2@unemi.edu.ec 
y rentabilidad resultaron óptimos en los tres últimos años analizados y en ISc, la tierra es propia. Los resultados permitieron entender la importancia de las sinergias que se generan con las interacciones en los sistemas productivos como estrategia de sostenibilidad.

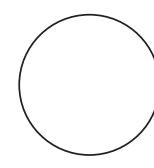

Palabras clave adicionales: agroecología; evaluación de indicadores; horticultura; plantas medicinales; sostenibilidad.

\section{ABSTRACT}

The objective of this research was to evaluate the sustainability of two agroecological production systems for vegetable and medicinal plants in a peri-urban area (NUDES-La Limonera) and an agricultural area (FIRP) in Venezuela. Descriptive observations were made for eleven months between 2014 and 2015. A sociodemographic questionnaire was applied, and sixteen sustainability indicators were quantified for the dimensions: ecological (IE), economic (IK), and sociocultural (ISc), with previously published methodologies. NUDES-La Limonera is part of a political project from the National Executive Branch, and its sustainability indicators reached less favorable levels, especially for EI (conservation of life in the soil and dependence on external inputs) and IK (food self-sufficiency, role of family labor, economic risk and diversity of products for sale). ISc contained indicators that showed greater strength (particularly the density of relationships with other groups); however, the satisfaction of basic needs, such as housing, was not met because the land is not owned (only guardianship and custody). The FIRP is part of an initiative of organized producers for the agricultural diversification of the Venezuelan Andes. It presented the most favorable values for sustainability in the three dimensions. It is worth mentioning that, for IK, productivity and profitability were optimal in the last three analyzed years and, for ISc, the land was owned. The results evidenced the importance of synergies that are generated within interactions in productive systems as a sustainability strategy.

Additional key words: agroecology; horticulture; medicinal plants; evaluation indicators; sustainability.

Fecha de recepción: 16-03-2018 Aprobado para publicación: 30-11-2018

INTRODUCCIÓN

La producción agropecuaria es un fenómeno complejo porque en su proceso intervienen las dimensiones ecológica, económica, sociocultural y política (Gliessman, 1998; Altieri, 1999; León 2014). Para que un sistema agroalimentario sea considerado sostenible, tiene que reunir cuatro criterios básicos: sostenibilidad ecológica, justicia social, viabilidad económica y aceptabilidad cultural (Sarandón y Flores, 2014; Argüello, 2015).

Uno de los enfoques de evaluación de la sostenibilidad agrícola incluye el uso de indicadores como una expresión de situaciones complejas, los cuales reflejan estados o tendencias de procesos relativamente complejos pero mostrados en una forma más entendible (Hayati et al, 2010). Los indicadores de sostenibilidad pueden medir variables tanto de naturaleza cuantitativa como cualitativa $y$, en términos generales, existen los indicadores de tendencia (aspectos dinámicos de la sostenibilidad en el tiempo) y los indicadores de estado que capturan la condición de los ecosistemas.

En estudios realizados por el Instituto de Investigaciones Agrícolas Venezolano, calcularon un índice de sostenibilidad que indicó que la agricultura venezolana en el año 1997 era ligeramente sostenible, con una tendencia al deterioro con el tiempo (Berroterán 
y Zink, 1997). Estos autores encontraron en su estudio que la sostenibilidad agrícola era fuerte hasta mediados de los años 70 y débil a partir de esa fecha. El motivo se lo atributen a: monocultivo, abandono de tierras deforestadas por degradación del suelo, baja eficiencia económica y bajos niveles de producción en relación con el potencial del cultivo, independientemente de los altos insumos incorporados al proceso productivo.

Esta situación se mantiene actualmente en Venezuela, pero los primeros 10 años del Siglo XXI se impuso un marco legal que favorece la institucionalización de la agroecología (Herrera et al., 2017). Actualmente es promovida, desde la academia y desde instituciones gubernamentales, entre algunos movimientos rurales y urbanos.

Un grupo de agricultores/as, para alcanzar sus objetivos productivos y socioeconómicos, decidió adoptar la agroecología como camino de producción y forma de vida. La literatura reporta que ya en el año 2008 había 74 comunidades productivas, distribuidas en 23 municipios (a nivel nacional) (Férgusson, 2011). Con más de 2.200 productores/as trabajando en alrededor de 1.200 parcelas (4.000 ha, aproximadamente) se han identificado más de 100 cultivos con prácticas agroecológicas.

De acuerdo con Núñez (2010) todas estas experiencias agroecológicas tienen diferentes niveles desarrollo y contribuyen a que unos 5.500 productores y productoras agroecológicas cubran unas 22.000 ha en diversos estados del país. Este colectivo tiene como horizonte el fortalecimiento de la soberanía alimentaria y han dado pauta para que, incluso, aparezca dicho término en la constitución, en un intento por garantizar que se cumpla el derecho a la alimentación (Ortega-Cerdà y Rivera-Ferre, 2010).

El objetivo de este trabajo fue evaluar la sostenibilidad agroecológica de dos de estos sistemas de producción agroecológica. De un espacio periurbano de Caracas: el Núcleo de Desarrollo Endógeno Socialista-La Limonera (NUDES-La Limonera) y la zona rural en el estado Mérida FIRP, ubicada en el Páramo Andino Venezolano. Se pretende, con este estudio, fortalecer y visibilizar propuestas de agroecología emergente y así ampliar información relevante sobre el tema, que actualmente presenta un vacío en Venezuela.

\section{MATERIALES Y MÉTODOS}

El trabajo se realizó en dos fincas consideradas por las asociaciones de productores locales como agroecológicas, cuyos propietarios/as aceptaron participar en la investigación durante el tiempo requerido. Los sistemas correspondieron al Núcleo de Desarrollo Endógeno Socialista La Limonera (NUDES-La Limonera), ubicado en la zona periurbana de Caracas y la FIRP, situado en la zona rural del estado Mérida, ambos sistemas en Venezuela. El levantamiento de la información se efectuó en el período octubre 2014 - agosto 2015.

Para evaluar cada sistema se empleó un cuestionario sociodemográfico con preguntas para las tres dimensiones de la agroecología (Ottmann, 2005). En la primera parte se indagó sobre datos generales referidos a la edad, género, escolaridad y estructura familiar. Así como, acerca de las Fincas, los rubros producidos, el destino (autoconsumo o venta) y los insumos requeridos para la producción (tanto químicos como biológicos) $\left(\mathrm{OSALA}^{1}\right)$.

La segunda parte del cuestionario se elaboró atendiendo a la propuesta de Masera et al. (2000), sobre atributos de la sostenibilidad que permiten hacer una mejor descripción de los sistemas. Se hicieron entrevistas estructuradas, las cuales fueron grabadas y se realizaron registros fotográficos de los predios. La información y los datos registrados se transcribieron, codificaron y ponderaron (Sarandón y Flores, 2014).

La observación directa en campo se realizó en tres estancias diferentes, a lo largo del período estudiado. Se organizaron reuniones y se impartieron talleres con personas que hacen vida dentro de cada sistema: como proveedores, redes de consumidores responsables, centros de investigación, universidades, entes gubernamentales y consejos comunales. Con la información obtenida de estas sesiones se realizó una matriz FODA, de la cual surgieron los criterios de diagnóstico MESMIS (Masera et al., 2000) para los criterios de diagnóstico, atributos de sostenibilidad, indicadores y sub-indicadores (Tab. 1).

\footnotetext{
http://www.osala-agroecologia.org/
} 


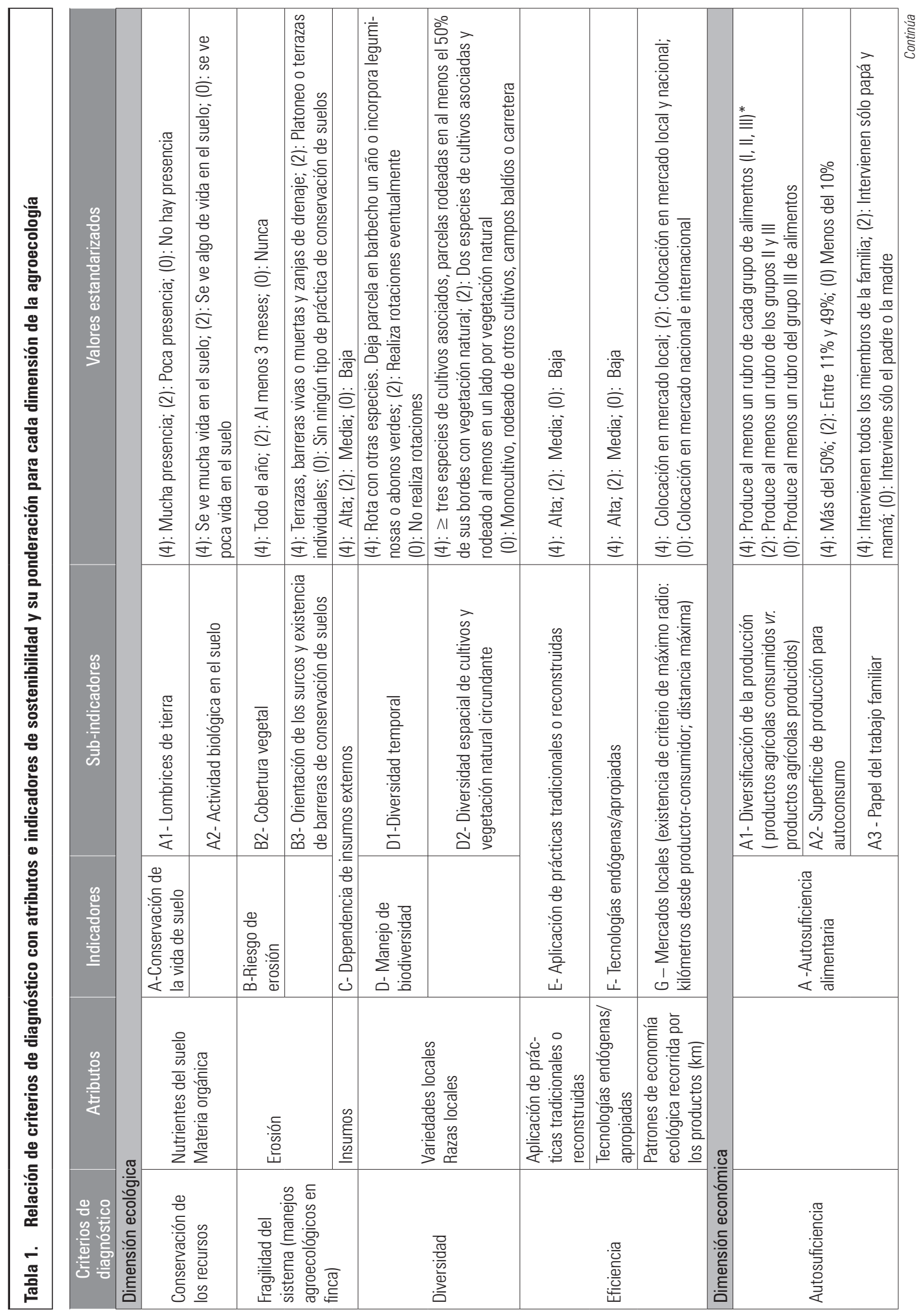




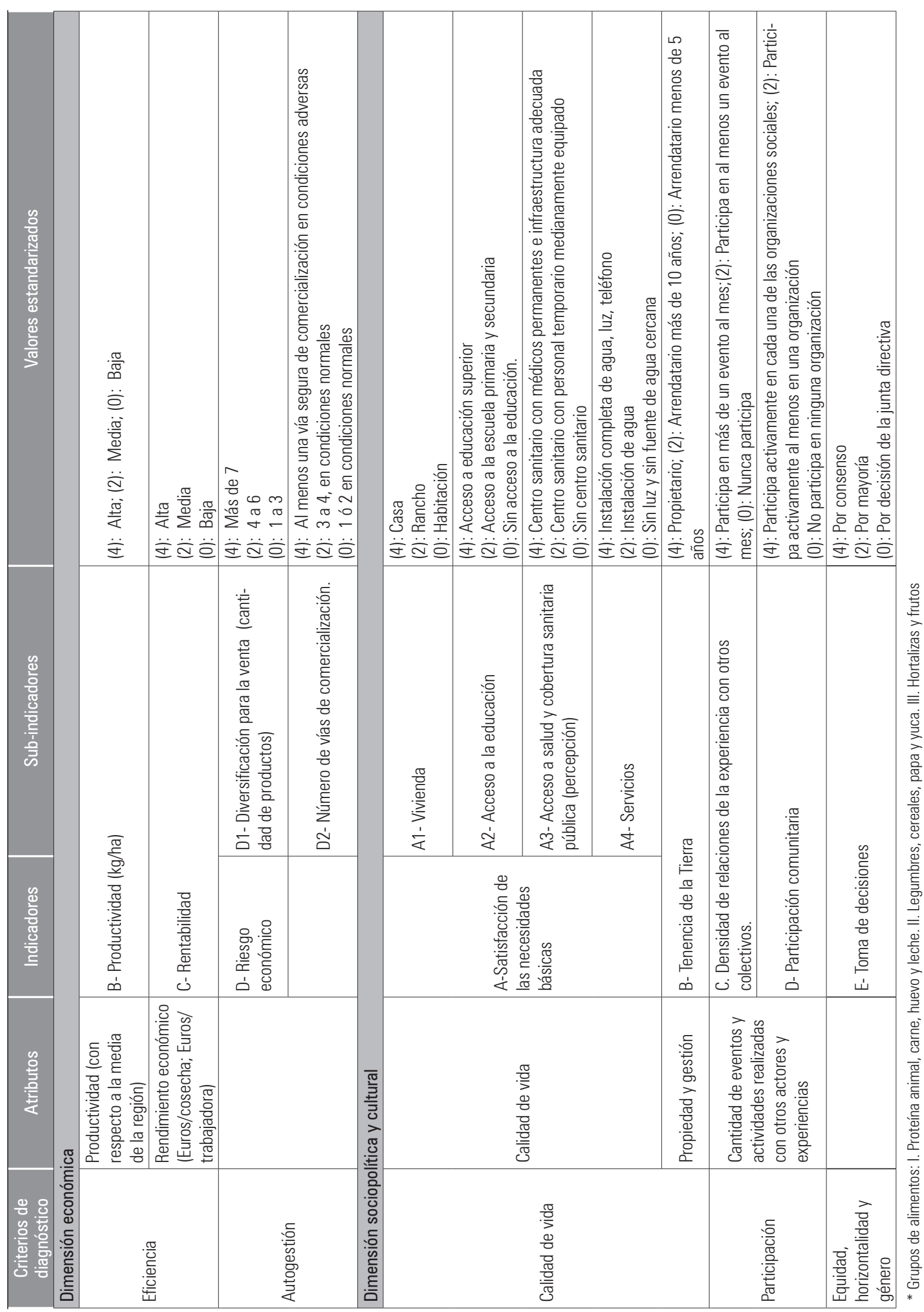


Para determinar el grado de cumplimiento para las diferentes dimensiones evaluadas, se calcularon a partir de las siguientes ecuaciones los índices ecológica (IE) (1); económica (IK) (2) y política-sociocultural (ISc) (3), como se describe a continuación:

$I E=$

$$
\begin{aligned}
& {[(A 1+A 2) / 2+(B 1+B 2) / 2+C} \\
& +(D 1+D 2) / 2+E+F+G] 7^{-1}
\end{aligned}
$$

donde IE es el índice ecológico, $A 1$ lombrices de tierra, $A 2$ actividad biológica en el suelo, $B 1$ cobertura vegetal, $B 2$ orientación de los surcos y existencia de barreras de conservación de suelos, $C$ dependencia de insumos externos, D1 diversidad temporal, D2 diversidad espacial de cultivos y vegetación natural circundante, $E$ aplicación de prácticas tradicionales o reconstruidas; $F$ tecnologías endógenas apropiadas y $G$ mercados locales

$I K=[(A 1+A 2+A 3) / 3+B+C+(D 1+D 2) / 2] 4^{-1}$

donde $I K$ es el índice económico, $A$ diversificación de la producción, $A 2$ superficie de producción para autoconsumo, $A 3$ papel del trabajo familiar, $B$ productividad (kg/ha); $C$ rentabilidad; $D 1$ diversificación para la venta (número de productos) y D2 número de vías de comercialización

$I S c=[(A 1+A 2+A 3) / 3+B+C+D+E] 6^{-1}$

donde $I S c$ es el índice sociocultural, $A 1$ vivienda, $A 2$ Acceso a la educación, $A 3$ acceso a la salud y cobertura sanitaria pública, $A 4$ servicios, $B$ tenencia de la tierra, $C$ densidad de relaciones con otros colectivos, $D$ participación comunitaria y $E$ toma de decisiones.

Siguiendo la propuesta de Sarandón y Flores (2014), se le otorgó el mismo peso a cada uno de estos indicadores y, así mismo, se calculó el índice de sostenibilidad general (ISGen) (4), es decir, se valoraron por igual cada una de las tres dimensiones. Este índice se describe como

$I S G e n=(I K+I E+I S c) 3^{-1}$

donde IE es el índice ecológico, IK índice económico y ISc índice sociocultural y político.

Para todos los indicadores se utilizó una escala de valoración 0,0-4,0 donde el primer valor corresponde al menos favorable y el último al óptimo de sostenibilidad (Sarandón y Flores, 2014).

\section{RESULTADOS Y DISCUSIÓN}

\section{Caracterización de los sistemas productivos}

NUDES-La Limonera. En el año 2005 se capacitó a un grupo de personas de costumbres urbanas con el fin de empoderarlos en su derecho a conformar asociaciones de carácter social y participativo, que podían ser cooperativas y otras formas asociativas, tal como lo manda el artículo 118 de la Constitución de la República Bolivariana de Venezuela (CRBV) (ANV, 1999). Ese año se constituyó la Cooperativa Agropecuaria La Limonera (CALL) conformada por 32 personas (NUDES La Limonera, 2005). El marco jurídico, y la iniciativa del Ejecutivo Nacional conocido como Misión Vuelvan Caras ${ }^{2}$ permitieron a la CALL recibir 194 ha de tierras en un área periurbana del municipio Baruta, en el área Metropolitana de Caracas. Del total de superficie, en 33 ha se consolidó el Núcleo de Desarrollo Endógeno Socialista La Limonera (NUDES La Limonera, 2005). La ocupación socio-territorial de La Limonera ha sido conflictiva debido a los intereses de los vecinos habitantes de las urbanizaciones adyacentes de la cooperativa Agroecológica La Limonera y del Estado (Fernández, 2013).

El NUDES-La Limonera basa su funcionamiento en cuatro ejes fundamentales: productivo, educativo y recreacional, asistencial y vivienda, como servicio público. Como fundamentos ideológicos consideran: satisfacer las necesidades "sentidas" del ser humano; integrar salud, vivienda, educación y trabajo; aprovechar los terrenos ociosos; extender sus beneficios a las comunidades aledañas; ser un modelo de desarrollo endógeno y ser autosostenible (NUDES La Limonera, 2005). Los NUDES, incluyendo La Limonera, son conceptualmente diversificados, pero en la mayoría de los casos están orientados a la actividad agrícola y la formación y producción agro-artesanal (Rojas y Pulido, 2009).

Dentro de las demarcaciones establecidas se reconoció "una zona de restauración ecológica para las áreas más degradadas, zonas para frutales y cultivos

\footnotetext{
2 La Misión Vuelvan Caras es un programa social impulsado por el gobierno bolivariano de Venezuela en el año 2003. Su objetivo es transformar el modelo económico imperante, basado en las leyes del mercado, en un modelo real de economía social, incorporando a la participación ciudadana. Complementándose con otras Misiones de la Educación como son Misión Robinson, Misión Ribas y Misión Sucre.
} 
hortícolas, zona de cría de animales y producción de materia orgánica, zona de acopio y procesamiento de alimentos, entre otras" (NUDES La Limonera, 2009).

Un objetivo fundamental de este proyecto político fue la construcción de un canal para promover la participación ciudadana dentro de los planes de producción agroalimentaria, en un espacio urbano. Este conjunto de objetivos, fundamentos ideológicos y ejes estratégicos es la conformación de lo que Gascón-Pérez (2014) han llamado democracia radical. Estos autores consideran que, más que orientaciones ideológicas cerradas para entender las relaciones sociales, son principalmente prácticas y lazos que centran su enfoque en la cooperación social y en la horizontalidad. Todo esto constituye un asiento para la construcción de vínculos sociales que conducen hacia la satisfacción, en conjunto, de las necesidades básicas; que integran desde abajo, en forma participativa, las dimensiones socioeconómicas, políticas y medioambientales.

En este sentido, el NUDES desarrolla un proyecto socio-productivo basado en el cultivo de hortalizas, cría de animales y un comedor comunitario que satisface la demanda de alimentos de los más necesitados en las zonas aledañas a La Limonera. Cada una de estas áreas ha sido identificada como un subsistema dentro del sistema total socio-productivo (Fig. 1).

Finca integral FIRP. Se encuentra ubicada en el municipio Rangel, del estado Mérida, el cual pertenece a los
Andes venezolanos y destaca por ser zona agrícola. Dentro de su diversa producción, sobresale la papa por su trascendencia como un hilo conductor de cohesión cultural entre los habitantes del Páramo Andino; también, por la relevancia socioeconómica (Romero, 2003).

Los productores pertenecen a una familia que vive en Mucuchíes, compuesto por seis hijos con edades comprendidas entre nueve y dieciocho años. El padre de familia, es miembro de Productores Integrales del Páramo (PROINPA), una asociación de agricultores que surge como una propuesta de desarrollo, impulsada por el Programa Andes Tropicales, en el año 2000. PROINPA es una experiencia piloto que establece estrategias productivas para incorporar principios de biodiversidad. Conjuga elementos tales como: organización, participación, capacitación de los productores, ética ecológica y tecnologías alternativas, en función de la economía social (Fuenmayor y Rodríguez, 2006).

Esta iniciativa surge de la necesidad de propiciar cambios en el modo de asumir y vivir la agricultura, impuesto por la corriente desarrollista del siglo XX, que se empezó a establecer a partir de los años sesenta, con las técnicas propuestas por la revolución verde y que en la región se caracterizó por: a) la intensificación de la producción en monocultivo de la papa; b) la importación de semillas de papa híbrida (desplazando a la semilla autóctona); c) el alto uso de insumos

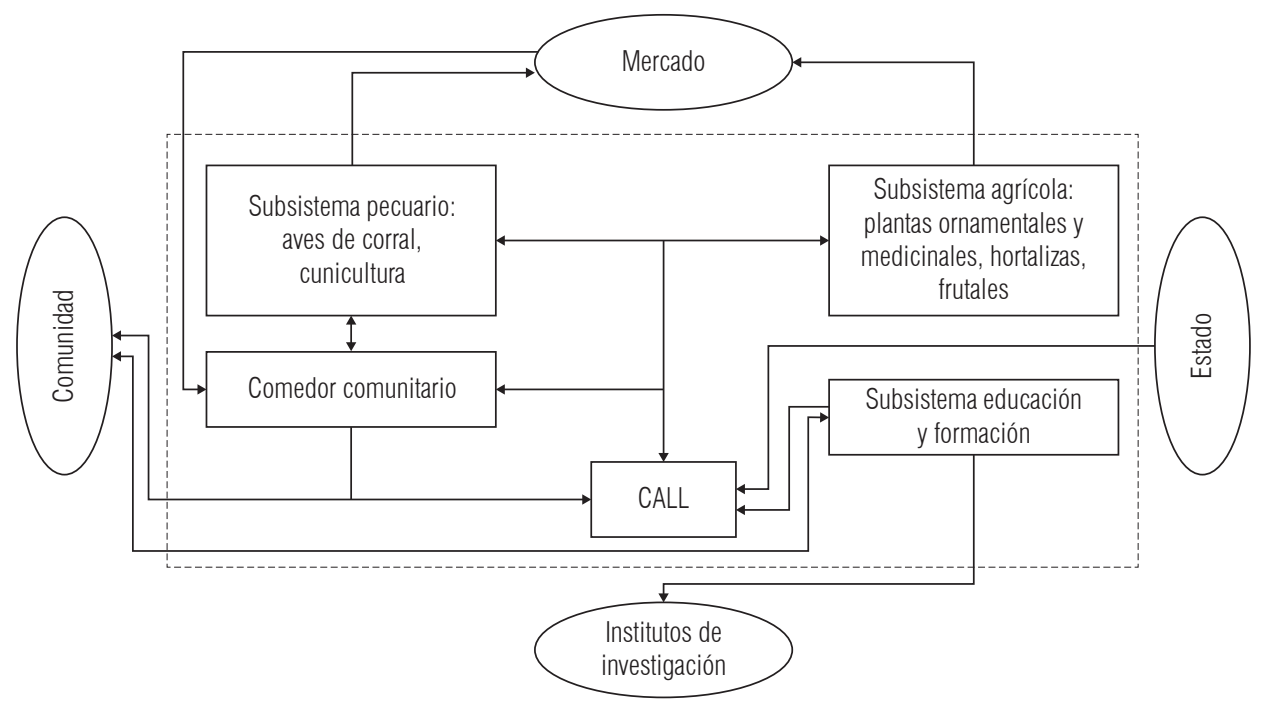

Figura 1. Diagrama de flujo de los subsistemas componentes del NUDES-La Limonera, Venezuela. 
químicos; d) el desplazamiento de la frontera agrícola hacia Parques Nacionales y d) el monopolio del mercado (Fuenmayor y Rodríguez, 2006).

De acuerdo con Sevilla (2011), la corriente científica convencional y su aplicación al concepto de desarro1lo, definió como "progreso" en las zonas rurales y campesinas a la homogeneización social, cultural, política y económica. Con ello causó la erosión del conocimiento local que se vino desarrollando con la interacción del ser humano y la naturaleza, en cada ecosistema específico. PROINPA emerge a contracorriente de esta definición de "progreso".

La experiencia de esta asociación de agricultores constituye un ejemplo en Venezuela, por sus aportes a la soberanía alimentaria. Se congregan en ella los y las productoras que reconocen la relación causa-efecto y que se atreven a construir una conexión entre el problema central de la homogeneización en exceso de la agricultura y su solución, es decir, se atreven a diversificar la producción (Romero y Romero, 2007). Es el caso de la FIRP.

Esta finca tiene 3 ha, una de ellas es para la producción de hortalizas y plantas medicinales, el resto es para mantener forraje para alimento de animales; utilizan la práctica de policultivos y asociación de cultivos simultáneos. En su sistema de manejo incorpora animales de corral y de pastoreo en los huertos medicinales (Tab. 2), el reciclaje de desechos, tanto de la finca como de la casa y del Mercado de Mérida, la sustitución de insumos químicos y la utilización de los mercados responsables para comercializar sus productos, se encuentran dentro de los subsistemas que conforman esta finca (Fig. 2).

\section{Tabla 2. Rubros producidos en cada una de las fincas tanto para el autoconsumo como para la venta}

\begin{tabular}{|c|c|c|c|}
\hline Origen & Autoconsumo & Venta & Nombre común de las especies \\
\hline \multicolumn{4}{|c|}{ NUDES-La Limonera } \\
\hline Vegetal & 7 & 7 & Ajo porro, cebollín, apio, cilantro, lechuga, papa, tomate, manzanilla y composta \\
\hline Animal & 4 & 3 & Conejos, gallinas y pollo \\
\hline Total & 11 & 10 & \\
\hline \multicolumn{4}{|c|}{ Finca Integral Rivera-Parra } \\
\hline Vegetal & 10 & 12 & $\begin{array}{l}\text { Ajenjo, alcachofa, arvejas, avena, caléndula, cebolla, cebollín, cilantro, guisantes, ha- } \\
\text { bas, linaza, manzanilla, marrubio, mejorana, papa, romero, ruda, tomillo, toronjil y trigo }\end{array}$ \\
\hline Animal & 7 & 4 & Gallinas, pollo, cerdos, leche, ovejos, pavos y queso \\
\hline Total & 17 & 16 & \\
\hline
\end{tabular}

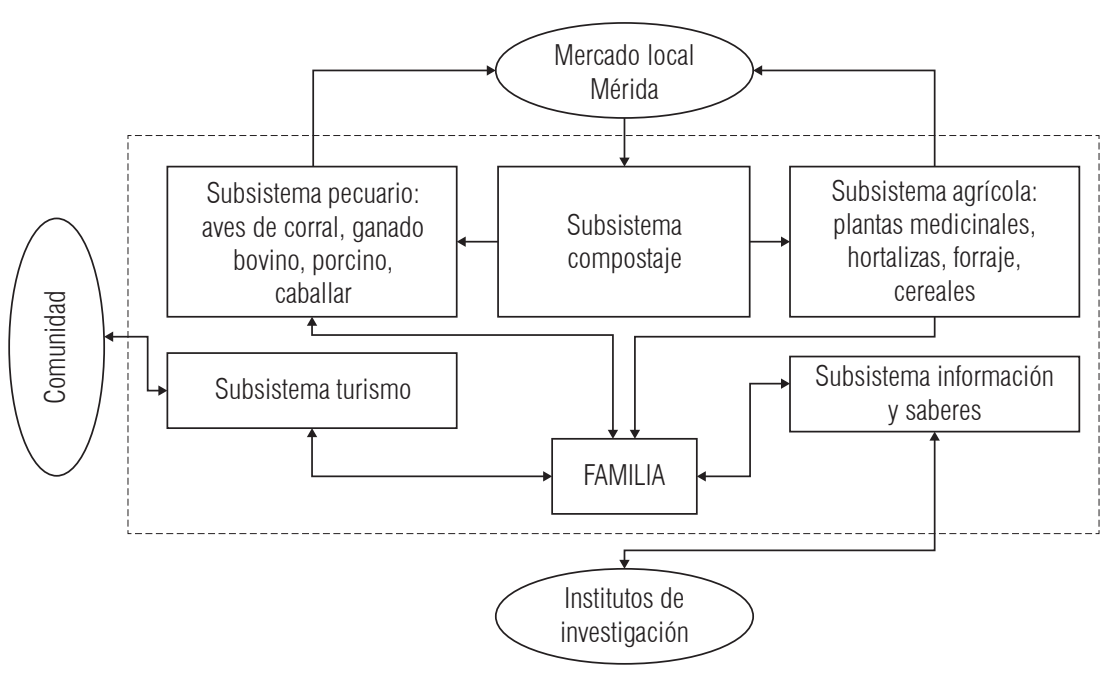

Figura 2. Diagrama de flujo de los subsistemas componentes de la finca integral FIRP (Merida, Venezuela). 


\section{Indicadores de sostenibilidad}

Los niveles de desempeño alcanzados por los diferentes indicadores de sostenibilidad permitieron realizar un diagnóstico para cada finca (Fig. 3).
Dimensión ecológica. En esta dimensión destacan los valores obtenidos en el NUDES-La Limonera para los indicadores: conservación de la vida en el suelo $(2,0)$ y dependencia de insumos externos $(2,0)$. Este valor medio obedece fundamentalmente a que el predio

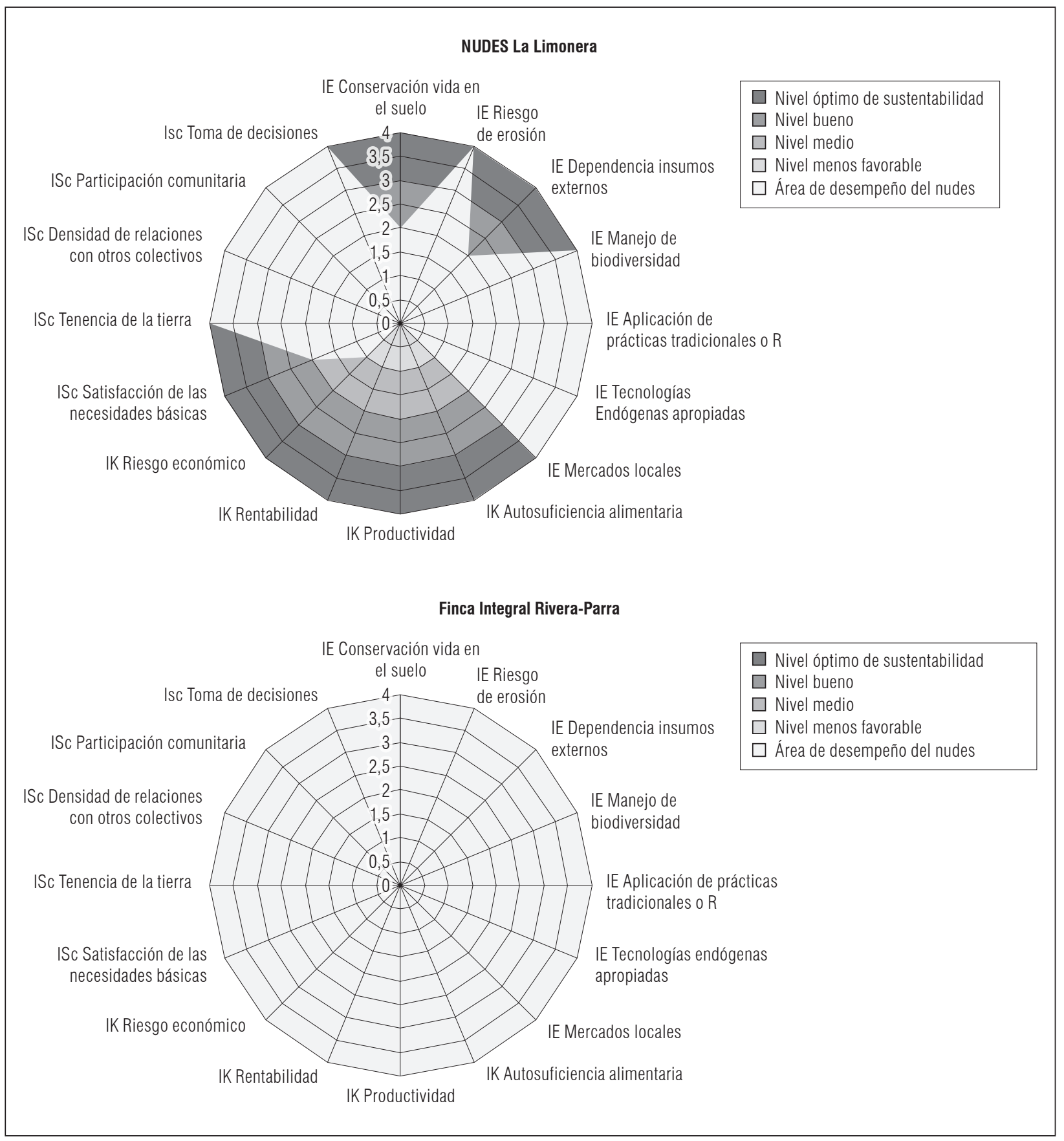

Figura 3. Niveles de desempeño obtenidos para los indicadores de sostenibilidad agroecológica del NUDES-La Limonera y la finca integral FIRP, Venezuela. 
no produce la mayoría de los insumos que requiere el comedor comunitario (Tab. 2); no obstante, sí se generan los insumos necesarios para la producción agrícola y pecuaria: como pueden ser el alimento para los animales, la composta y las semillas. Quiere decir que el resto de los insumos necesarios para satisfacer la demanda del comedor comunitario debe ser comprado en los mercados locales.

Cuando comenzaron la experiencia de producción, aplicaban agroquímicos para las hortalizas como ajo porro (Allium ampeloprasum L.), cebolla (Allium cepa L.), cebollín (Allium schoenoprasum L.), célery (Apium graveolens L.), cilantro (Coriandrum satium L.), lechuga (Lactuca sativa L.) y tomate (Solanum lycopersicum L.) y plantas medicinales como manzanilla (Chamaemelum nobile (L.) All.), marrubio (Marrubium vulgare L.), mejorana (Origanum majorana L.), ruda (Ruta graveolens L.), tomillo (Thymus vulgaris L.), toronjil (Melissa officinalis L.) y romero (Rosmarinus officinalis L.). Posteriormente, acompañados por investigadores y colaboradores externos, incursionaron en prácticas agroecológicas. Esto disminuyó el uso de insumos externos y les ayudó a construir un sistema integrado que incorpora los residuos en el proceso de producción $\mathrm{y}$, como consecuencia, disminuye costos.

Es importante, así mismo, la transformación que hacen de los productos agrícolas en el comedor, para ofrecer un servicio social. Esa es la razón por la que indicadores como aplicación de prácticas tradicionales/ reconstruidas $(4,0)$ y tecnologías endógenas apropiadas $(4,0)$, hayan resultado con valores óptimos de sostenibilidad. La literatura reporta que estas prácticas juegan un papel importante en la sostenibilidad agroecológica de los sistemas productivos porque disminuyen la dependencia del campesinado y conserva y mejora los recursos naturales (Van Driesche y Hoddle, 2007; Altieri y Nicholls, 2009).

Respecto a la Finca Integral FIRP, todos los indicadores de esta dimensión mostraron el valor óptimo de sostenibilidad. La finca integral genera insumos que son necesarios para la producción: mantienen permanentemente un cultivo de pastos que alimenta el ganado; el estiércol es insumo para la composta, con ella fertilizan el suelo (Tab. 2; Fig. 2). También, han adoptado tecnologías que les permiten ser más independientes de los factores externos. Por ejemplo: la máquina de secado de plantas medicinales es un apoyo para mejorar el almacenaje y la conservación de la producción. Estás prácticas propenden por la sostenibilidad del sistema (Van Driesche y Hoddle, 2007; Altieri y Nicholls, 2009).
Dimensión económica (IK). En el NUDES-La Limonera el indicador autosuficiencia alimentaria refleja la situación que ellos viven, aun cuando no alcanzan niveles deseables de autosuficiencia alimentaria. Una parte de la producción satisface la demanda del comedor comunitario y otra destinada a la venta.

Para el sub-indicador papel del trabajo familiar, los resultados fueron menos favorables a la sostenibilidad (0) pues que los familiares de los integrantes de la cooperativa no participan en las labores de producción.

Al revisar la contabilidad se observó una reducción en la productividad de hortaliza en los últimos años pasando de 5 t en el 2013, 1,5 t 2014 y >1 t 2015. Estas cifras son explicadas al déficit de mano de obra que demandan dichas labores agrícolas. La mayoría de los integrantes realizan actividades paralelas fuera del núcleo con el propósito de obtener mayores ingresos.

El sub-indicador diversidad de productos para la venta $(2,0)$, influenció negativamente al indicador riesgo económico (1,0); al tener un solo agente de compras (entidades gubernamentales) se limita el beneficio económico al fijar el precio de compra por debajo del establecido en el mercado local. Esto influye negativamente el resultado final de indicador riesgo económico $(1,0)$.

En caso contrario, la mayoría de los indicadores de la finca integral FIRP presentaron valores óptimos a la sostenibilidad. Por ejemplo, el indicador autosuficiencia alimentaria arrojó el valor máximo $(4,0)$; la finca produce por lo menos un rubro de cada uno de los grupos de alimentos para las categorías del grupo I: leche, huevos y carne (ovejas, cerdos, vacas y gallinas), grupo II: papas y cereales y grupo III: hortalizas (Tab. 2).

Todos los miembros de la familia intervienen en la producción, razón por la cual el indicador papel del trabajo familiar $(4,0)$ obtuvo el máximo valor. Los miembros participan activamente en épocas de mayor faena (la cosecha), sin interferir con las labores escolares. Los indicadores, productividad y rentabilidad, resultaron con valores óptimos $(4,0)$ y se ha mantenido así durante los últimos tres años (según registros). La diversidad de la producción es un factor importante que contribuye al mantenimiento económico evitando el riesgo y maximizando la rentabilidad; perciben ingresos por la venta de queso, carne de oveja y lana. También obtienen ingresos adicionales por turismo al prestar servicios de guía y hospedaje en temporada 
alta. Realizan ventas directas, sin intermediarios. Practican intercambios o trueque con algunas comunidades (semillas y, en menor medida, frutales o hierbas medicinales).

Los sistemas productivos estudiados aportan múltiples elementos en esta dimensión económica de la agroecología, la literatura reporta que estas prácticas aportan en la construcción de la soberanía alimentaria (Toledo y Barrera, 2009). Los resultados presentan saldos positivos en esta dimensión, tanto al interior de cada sistema investigado como en sus áreas de influencia.

Dimensión política sociocultural (ISc). Los miembros del NUDES-La Limonera ha logrado reivindicaciones importantes como guardia y custodia de tierras, el apoyo económico del gobierno para la construcción de casas de cultivo, el caso de la vivienda es un tema pendiente por resolver ya que la mayoría no vive dentro del predio. "La vivienda como servicio público es uno de los ejes fundamentales del proyecto NUDES-La Limonera. Sus integrantes esperaban que se les asignara una vivienda en el complejo habitacional que otorgó el gobierno. Sin embargo, algunos miembros fueron excluidos de la adjudicación de apartamentos que se realizó en el año 2011 en la Ciudad Socialista La Limonera, un diseño urbanístico concebido con casi mil apartamentos financiados por el gobierno Nacional (Fernández, 2013).

El indicador densidad de relaciones con otros colectivos, alcanzó el valor óptimo a la sostenibilidad debido al intercambiado experiencias con otros NUDES a nivel nacional durante los últimos ocho años. Esto resulta ser una fortaleza porque el intercambio de experiencias incrementa la posibilidad de mejorar sus sistemas de manejo. En el NUDES-La limonera hay un centro de desarrollo y formación sociopolítica donde realizan talleres con fines educativos y encuentros con fines de divulgación acerca de los beneficios de las actividades socio productivas y auto gestionadas desarrolladas durante los últimos años.

El programa "rescate de niños de calle", imparte talleres de formación con miras a la incorporación de actividades de producción agrícola: selección de las semillas, siembra y cosecha.

La organizan de comisiones de trabajo es un logro importante para ellos, porque según las entrevistas, "les ha permitido adecuarse a las capacidades y vocaciones de cada quien", lo que los hace trabajar con "entusiasmo y amor, apoyados en la confianza y el respeto mutuo". En cuanto a la toma de decisiones, se toman por consenso, sin predominio de género, nivel educativo, clase social, ni ningún otro factor de discriminación.

Cuando se indagó en la entrevista sobre las nuevas formas de cooperación y corresponsabilidad, żcómo se perciben como actores?, respondieron: "viviendo en espacios urbanos, nos interesa producir para cuidar la salud y para cuidar y recuperar el suelo". Ellos se conciben a sí mismos como actores de cambio social: "creemos en el socialismo, creemos que es importante proliferar y conservar estos espacios, para gestar una conciencia de cambio que derive en un desarrollo comunitario endógeno".

Partiendo de ese imaginario, dentro de las actividades socioculturales y sociopolíticas, los integrantes del NUDES-La Limonera perciben que tienen la oportunidad de "construir un modelo de país más justo, más respetuoso con el medioambiente y con los recursos naturales". Este imaginario urbano con concepciones de autosuficiencia alimentaria mediante la producción agropecuaria, se indagó sobre żqué falta para llegar a ese imaginario? Contestaron: "falta organización, planificación y personas que estén comprometidas con el desarrollo del área socio-productiva de este proyecto, que dependan directamente de su éxito para su sustento".

La FIRP, en esta dimensión económica, resultó con valores óptimos de sostenibilidad, para todos los indicadores. La familia ha tenido acceso a los recursos, todo el predio es de su propiedad, esto ha influido para que los indicadores de satisfacción de las necesidades básicas y tenencia de la tierra resultaran con el máximo valor en la escala (4,0 para ambos). La vivienda se encuentra en buen estado y tiene todos los servicios públicos; ellos la describen como "el paraíso terrenal". Todos los integrantes de la familia desempeñan un rol importante en la producción. Se destaca el el papel de la señora de la casa, en lo que se denomina trabajo productivo y reproductivo (Benería, 2006). También es la administradora de los rubros relacionados con el turismo como el hospedaje. Todos los miembros de la familia participan en las decisiones que se toman por consenso.

En cuanto a los indicadores, acceso a la educación y a la salud, el resultado fue favorable a la sostenibilidad. En la zona se encuentran servicios tales como universidades, hospital, CDI (Centro de Diagnóstico Integral) y CRI (Centro de Rehabilitación Integral), estos últimos equipados adecuadamente con tecnología de última generación. 
Respecto a los indicadores de participación comunitaria y densidad de relaciones con otros colectivos, desde hace más de siete años se vienen desarrollando intercambios con otros NUDES del país. Por otro lado, mantienen un constante intercambio con el resto de los miembros de la Asociación Mano a Mano y de PROINPA. Hay que destacar la interacción que mantienen con institutos de investigación como la Universidad de Los Andes, Universidad del Zulia y Instituto Nacional de Investigaciones Agrícolas (INIA), porque contribuyen a generar y difundir un conocimiento que aporta a la soberanía alimentaria.

En lo referente al imaginario sobre la ruralidad y agricultura campesina, se encuentran testimonios como "yo vivo en el campo y vivo del campo, somos capaces de producir nuestros alimentos y de vivir dignamente". Son consumidores de productos agroecológicos producidos en la zona y de temporada, lo hacen por conciencia ambiental y salud. Muchos productos los adquieren a través de la Asociación Mano a Mano.

La proliferación de estos espacios permiten la participación y organización, y es uno de los resultados de esta dimensión sociocultural, esto para Cuéllar y Sevilla (2009) es de suma importancia porque las consideran como acciones que conllevan a la autogestión de los procesos de circulación, y esto ayuda a una transformación endógena desde lo social, lo económico y lo político.

Un pilar de la soberanía alimentaria lo constituye el acceso y control, de manera equitativa y sostenible en el manejo de los recursos naturales (Ottmann, 2005; Ortega-Cerdà y Rivera-Ferre, 2010), en ambos casos se detecta una fortaleza en cuanto al acceso de los recursos; sin embargo el caso de NUDES-La Limonera, aunque ya están en posesión de una tierra que es de propiedad colectiva, hace falta tomar acción en el área socio-productiva para ir hacia la producción de alimentos que permita el auto sustento de todas las personas que hacen vida dentro este núcleo de desarrollo.

\section{CONCLUSIONES}

El análisis de las experiencias y la visión integral del estudio ayudó a establecer relaciones entre las distintas dimensiones de la agroecología, tanto para lo urbano como en lo rural. Este enfoque sistémico permitió comprender mejor el fenómeno de la producción agroalimentaria con prácticas agroecológicas en ambas experiencias.
En el sistema del NUDES-La Limonera, el estudio reveló deficiencias en las prácticas de manejo de los recursos naturales, aspectos acertados en la participación comunitaria y la relación con otros colectivos agroecológicos.

La comparación de los sistemas agro-productivos (rural y urbano) y sus interrelaciones, más que constatar el grado de dependencia de los insumos externos o concentrarse en la especificación y diversificación del sistema, permite comprender lo fundamental que resulta para el sistema las sinergias que se generan a partir de sus interacciones. Esto contribuye a mantener el sistema en producción constante y sin afectar el equilibrio. Es una estrategia para la sostenibilidad y la productividad en el largo plazo. La identidad cultural estrechamente ligada al consumo y la producción, como pudo evidenciarse en la Finca Integral FIRP, es otro factor que contribuye a la sostenibilidad.

La integralidad del modelo de evaluación contempló el estudio de cada dimensión de la producción agroalimentaria, esto permitió un conocimiento más amplio de los componentes del sistema y la naturaleza y características propias: procesos y productos, formas de manejo, la relación entre ellos y de ellos con el contexto. La metodología permitió reconocer los avances hacia la sostenibilidad de tales sistemas, tanto en el ámbito rural como en el urbano.

Además, permitió detectar los procesos que necesitan un cambio en la estrategia de manejo: en el NUDES-La Limonera, el riesgo económico que representa para el sistema tener un solo comprador, que además es quien fija el precio de compra. También permite reconocer la importancia de articular los procesos productivos, con el trabajo colectivo y formas de producción alimentaria más sostenibles y vinculadas con la identidad cultural de sus integrantes. En cuanto a FIRP permitió constatar la eficiencia productiva alcanzada por implementar prácticas de manejo con principios agroecológicos de diversidad, reciclaje de nutrientes, autogestión y participación comunitaria.

\section{AGRADECIMIENTOS}

Este trabajo no hubiera sido posible sin el apoyo incondicional de los miembros de la comunidad NUDES-La Limonera y de la finca integral FIRP. Para ellos nuestro agradecimiento. 
Conflicto de intereses: el manuscrito fue preparado y revisado con la participación de los autores, quienes declaran no tener algún conflicto de interés que coloquen en riesgo la validez de los resultados aquí presentados.

\section{REFERENCIAS BIBLIOGRÁFICAS}

Altieri, M. 1999. Agroecología: bases científicas para una agricultura sostenible. Nordan-Comunidad, Montevideo.

Altieri, M. y C. Nicholls. 2009. Conversión agroecológica de sistemas convencionales de producción: teoría, estrategias y evaluación. Ecosistemas 16, 3-13.

ANV, Asamblea Nacional de Venezuela. 1999. Constitución de la República Bolivariana de Venezuela. República de Venezuela. Gaceta Oficial 5.453 Extraordinario de fecha 24 de marzo de 2000. Caracas.

Argüello, H. 2015 Agroecology: scientific and technological challenges for agriculture in the 21st century in Latin America. Agron. Colomb. 33(3), 391-398.

Benería, L. 2006. Trabajo productivo/reproductivo, pobreza y políticas de conciliación. Nómadas 24, 8-21.

Berroterán, J. y J. Zinck. 1997. Indicators of agricultural sustainability at national level. A case study of Venezuela. ITC-Journal 1997-3/4.

Cuéllar, M. y E. Sevilla. 2009. Aportando a la construcción de la Soberanía Alimentaria desde la Agroecología. Ecol. Pol.: Cuad. Debate Int. 38, https://www.ecologiapolitica.info/?p=4848; consultado; junio de 2018

Férgusson, A. 2011. Agroecología para docentes de América Latina. Ediciones Universidad Bolivariana de Venezuela, Caracas.

Fernández, B. 2013. La integración socio-territorial de los sectores populares a la ciudad: un proceso conflictivo. Caso del nuevo urbanismo popular La Limonera. Rev. Venez. Anal. Coyunt. 19(2), 129-157

Fuenmayor, N. e Y. Rodríguez. 2006. Aportes de la economía social al desarrollo sostenible. El caso de la Asociación de Productores Integrales del Páramo en el Estado Mérida, Venezuela. Rev. Venez. Cienc. Soc. 10(1), 144-160.

Gascón-Pérez, L. 2014. Democracia radical, entre la crítica y el nihilismo: un abordaje de la propuesta desde el diálogo entre Ernesto Laclau y Slavoj Žižek. Rev. Mex. Cienc. Polít. Soc. 59(221), 121-144. Doi: 10.1016/ S0185-1918(14)70818-9

Gliessman, S. 1998. Agroecología: procesos ecológicos en agricultura sostenible. CATIE, Costa Rica.

Hayati, D., Z. Ranjbary E. Karami. 2010. Measuring agricultural sustainability. pp. 73-100. En: Lichtfouse L. (ed.)
Biodiversity, biofuels, agroforestry and conservation agriculture. Springer, Dordrecht, The Netherlands.

Herrera, F., O. Domené-Painenao y J. Cruces. 2017. The history of agroecology in Venezuela: a complex and multifocal process. Agroecol. Sust. Food 41(3), 401-415. Doi: 10.1080/21683565.2017.1285842

León, T. 2014. La perspectiva ambiental de la agroecología: la ciencia de los agroecosistemas. Instituto de Estudios Ambientales, Universidad Nacional de Colombia, Bogotá.

Masera, O., M. Astier y S. López-Ridaura. 2000. Sostenibilidad y manejo de los recursos naturales, el marco de evaluación MESMIS. Ediciones MundiPrensa, México.

NUDES La Limonera. 2005. Documento constitutivo NUDES, La Limonera. Caracas [Documento sin editar].

NUDES La Limonera. 2009. Normas de la Escuela de Formación Samuel Robinson. Caracas [Documento sin editar].

Núñez, M. 2010. Venezuela ecosocialista: un debate pendiente. Portatítulo, Caracas.

Ortega-Cerdà, M. y G. Rivera-Ferre. 2010. Indicadores internacionales de soberanía alimentaria. Nuevas herramientas para una nueva agricultura. Rev. Iberoam. Econ. Ecol. 14, 53-77.

Ottmann, G. 2005. Agroecología y sociología histórica desde Latinoamérica: Elementos para el análisis y potenciación del movimiento agroecológico: el caso de la provincia argentina de Santa Fe. Mundi-Prensa; UCO; PNUMA, Madrid.

Rojas, J. y N. Pulido, 2009. Estrategias territoriales recientes en Venezuela: ¿̇Reordenación viable de los sistemas territoriales o ensayos de laboratorio? EURE 35(104), 77-100. Doi: 10.4067/S0250-71612009000100004

Romero, L. 2003. Hacia una nueva racionalidad socio-ambiental en los Andes Paperos de Mérida. ¿̇De qué depende? Fermentum 13(36), 55-72.

Romero, L. y R. Romero. 2007. Está germinando una nueva propuesta en agricultura. Agroecología en los Andes venezolanos. En: http://www.saber.ula.ve/ bitstream/123456789/21456/2/articulo10.pdf; consultado: agosto de 2017.

Sarandón, S. y C. Flores. 2014. Agroecología: bases teóricas para el diseño y manejo de agroecosistemas sostenibles. Universidad Nacional de La Plata, La Plata, Argentina.

Silva-Laya, S., S. Pérez-Martínezy L. Ríos-Osorio. 2016. Evaluación agroecológica de sistemas hortícolas en dos zonas del oriente antioqueño. Rev. Colomb. Cienc. Hort. 10(2), 355-365. Doi: 10.17584/rcch.2016v10i2.5752

Sevilla, E. 2011. De la sociología rural a la agroecología. Icaria, Barcelona, España. 
Toledo, V. y N. Barrera. 2009. La memoria biocultural. Barcelona, Icaria, España.

Van Driesche, R. y M. Hoddle. 2007. El papel de la ecología de poblaciones y de los modelos de población en el control biológico. pp. 153-178. En: Van Driesche, R., M. Hoddle y T.D. Center (eds.) Forest health technology enterprise team: Control de plagas y malezas por enemigos naturales. USDA, Washington DC. 